\title{
Continuous-matrix-product-state solution for the mixing-demixing transition in one-dimensional quantum fields
}

\author{
Fernando Quijandría ${ }^{1}$ and David Zueco ${ }^{1,2}$ \\ ${ }^{1}$ Instituto de Ciencia de Materiales de Aragón and Departamento de Física de la Materia Condensada, \\ CSIC-Universidad de Zaragoza, Zaragoza E-50012, Spain \\ ${ }^{2}$ Fundación ARAID, Paseo María Agustín 36, Zaragoza 50004, Spain
}

(Received 2 August 2015; published 28 October 2015)

\begin{abstract}
We solve the mixing-demixing transition in repulsive one-dimensional Bose-Bose mixtures. This is done numerically by means of the continuous matrix product states variational ansatz. We show that the effective low-energy bosonization theory is able to detect the transition whenever the Luttinger parameters are exactly computed. We further characterize the transition by calculating the ground-state energy density, the field-field fluctuations, and the density correlations.
\end{abstract}

DOI: 10.1103/PhysRevA.92.043629

PACS number(s): 03.75.Hh, 67.85.Fg, 05.30.Rt, 03.65.Ca

\section{INTRODUCTION}

Understanding the physics of strongly correlated manybody systems is a formidable task, both in the lattice and in the continuum [1]. There is a fruitful synergy between condensed matter, high-energy physics or quantum chemistry, and the quantum information community. Ideas such as tensor network states [2] or quantum simulations [3] are pursuing the goal of understanding phases and dynamics beyond the paradigm of perturbative theories.

One-dimensional (1D) many-body systems are a good example of this cooperation. Well-established theoretical techniques as bosonization [4] are complemented with the density matrix renormalization group and matrix product states (MPSs) [5,6] in the lattice and more recently, continuous matrix product states (cMPSs) in the continuum [7]. Ultracold gases are a paradigmatic example of experiments realizing one dimensional quantum fields [8-10]. Experiments and simulations in one dimension are perfect test beds since each of them can be used for benchmarking the other [11-14].

Of special relevance for this work is the cMPS formalism. Introduced by Verstraete and Cirac [7], these states constitute a variational class for the efficient simulation of quantum field theories that does not rely on a space discretization. The ansatz has proven to be efficient for computing the ground state, dispersion relation [15], and quantum evolution [16] of nonrelativistic theories. In addition, introducing a suitable regularization prescription, it has also been applied to the study of certain relativistic phenomena [17]. Finally, the cMPS ansatz has been already tested for Bose-Bose [18] and Fermi-Fermi [19] mixtures, both in nonrelativistic setups.

In this work we will restrict ourselves to Bose-Bose mixtures [20,21]. Concretely, we aim to characterize the mixing-demixing phase transition occurring in repulsive bosonic mixtures via cMPS. Here the competition between the intra- and interspecies couplings leads to the formation of two different phases. The miscible, where the two gases coexist, and the immiscible, where the two gases separate from each other [22]. This transition has been studied within different approaches [23-27], but its numerical simulation in the continuum has been elusive.

The paper is organized as follows. In Sec. II we review the phase transition and discuss the different approaches used to characterize it. In Sec. III we briefly introduce the multispecies cMPS formalism. In Sec. IV, by using the already introduced cMPS approach, we characterize the mixing-demixing transition by means of the bosonization description and by calculating the ground state properties. Finally, in Sec. V we summarize our results.

\section{THE PHASE TRANSITION}

Two 1D bosonic gases interacting via a quartic contact potential $(\hbar=2 m=1)$ are described in second quantization with the Hamiltonian

$$
\begin{aligned}
\hat{H}= & \sum_{\alpha=1}^{2} \int_{0}^{L} d x \partial_{x} \hat{\psi}_{\alpha}^{\dagger}(x) \partial_{x} \hat{\psi}_{\alpha}(x) \\
& +\sum_{\alpha, \beta=1}^{2} g_{\alpha \beta} \int_{0}^{L} d x \hat{\psi}_{\alpha}^{\dagger}(x) \hat{\psi}_{\beta}^{\dagger}(x) \hat{\psi}_{\beta}(x) \hat{\psi}_{\alpha}(x) .
\end{aligned}
$$

Here $\hat{\psi}_{\alpha}^{\dagger}(x)\left[\hat{\psi}_{\alpha}(x)\right]$ are the bosonic field operators which create (annihilate) bosonic particles of species $\alpha$ at the position $x \in[0, L]$. They satisfy the commutation relations: $\left[\hat{\psi}_{\alpha}(x), \hat{\psi}_{\beta}^{\dagger}\left(x^{\prime}\right)\right]=\delta_{\alpha \beta} \delta\left(x-x^{\prime}\right)$ and $\left[\hat{\psi}_{\alpha}(x), \hat{\psi}_{\beta}\left(x^{\prime}\right)\right]=$ $\left[\hat{\psi}_{\alpha}^{\dagger}(x), \hat{\psi}_{\beta}^{\dagger}\left(x^{\prime}\right)\right]=0$. In this work, we want to characterize numerically the mixing-demixing transition occurring in mixtures whenever two different repulsive bosonic species are trapped together. We will consider the case where the participating species are nonconvertible, i.e., the individual particle densities $\rho_{0 \alpha}$ of each bosonic species are conserved separately $\left[\rho_{0 \alpha}=\left\langle\hat{\psi}_{\alpha}^{\dagger}(x) \hat{\psi}_{\alpha}(x)\right\rangle ;\langle\rangle\right.$ means averaging over the ground state of (1)]. We will also restrict to the symmetric case $\rho_{01}=$ $\rho_{02}=\rho_{0}, g_{11}=g_{22}=c>0$, and $g_{12}=g_{21}=g / 2>0$.

The mixing-demixing transition has been broadly studied analytically; see, e.g., Refs. [23,24,26,28-30]. The phase separation, which lies on the competition between the repulsion strengths $c$ and $g$, can be understood on several grounds. The simplest approach considers a mean-field treatment. Here the interaction term can be seen as a quadratic form of the densities. The latter is positive defined as long as $g<2 c$. When the positivity condition is violated $(g \geqslant 2 c)$ an instability occurs. In more than one dimension, both species must separate in order to make the overlap integral zero, i.e., minimizing the 
repulsive interaction which yields the instability. In this phase $(g \geqslant 2 c)$ both species are immiscible as observed by resolving the spatial density profiles in the trap [22]. In one dimension, there is no possibility of spatial separation. The interspecies fluctuations, $\left\langle\hat{\psi}_{1}^{\dagger}(x) \hat{\psi}_{2}^{\dagger}(x) \hat{\psi}_{1}(x) \hat{\psi}_{2}(x)\right\rangle$ in Eq. (1), are zero in the ground state.

One-dimensional systems are somehow special. Their confinement provides an enhancement of the collective behavior, leading to the Luttinger liquid universality class in the lowenergy or long-wavelength sector $[4,31,32]$. This regime is described by introducing the bosonic operators $\hat{\phi}_{\alpha}$ and $\hat{\theta}_{\alpha}$ in terms of which we rewrite the field operators $\hat{\psi}_{\alpha}(x)=\left(\rho_{0 \alpha}-\right.$ $\left.\partial_{x} \hat{\phi}_{\alpha}(x) / \pi\right)^{1 / 2} \sum_{p=-\infty}^{+\infty} \mathrm{e}^{i p\left(\pi \rho_{0 \alpha} x-\hat{\phi}_{\alpha}(x)\right)} \mathrm{e}^{i \hat{\theta}(x)}$. This is nothing but the harmonic fluid approach treatment best known in the literature as bosonization [33]. Note that for high enough values of $p$, the exponential terms oscillate very fast and rapidly average to zero. Therefore, in order to obtain the low-energy effective Hamiltonian, we should keep only a few relevant terms. This leads to

$$
\begin{aligned}
2 \pi \hat{H}_{\mathrm{eff}}= & \int d x \sum_{\alpha=1}^{2}\left[\frac{v_{\alpha}}{K_{\alpha}}\left(\partial_{x} \hat{\phi}_{\alpha}\right)^{2}+v_{\alpha} K_{\alpha}\left(\partial_{x} \hat{\theta}_{\alpha}\right)^{2}\right] \\
& +\int d x\left\{2 g_{x} \partial_{x} \hat{\phi}_{1} \partial_{x} \hat{\phi}_{2}+g_{c} \cos \left[\left(2\left(\hat{\phi}_{1}-\hat{\phi}_{2}\right)\right]\right\} .\right.
\end{aligned}
$$

This long-wavelength description is fully characterized by the dimensionless parameters $K_{\alpha}$, the velocities $v_{\alpha}$, and the coupling strengths $g_{x}$ and $g_{c}$ (Luttinger parameters). For the symmetric case considered here, we have that $v_{1}=v_{2}=v$ and $K_{1}=K_{2}=K$. This model can be easily decoupled by introducing the normal modes $\hat{\phi}_{ \pm}=1 / \sqrt{2}\left(\hat{\phi}_{1} \pm \hat{\phi}_{2}\right) \quad$ and $\quad \hat{\theta}_{ \pm}=1 / \sqrt{2}\left(\hat{\theta}_{1} \pm \hat{\theta}_{2}\right)$. In terms of them, the low-energy Hamiltonian reads $2 \pi \hat{H}_{\text {eff }}=\int d x \sum_{\nu= \pm}\left[\frac{v_{v}}{K_{v}}\left(\partial_{x} \hat{\phi}_{\nu}\right)^{2}+v_{v} K_{v}\left(\partial_{x} \hat{\theta}_{\nu}\right)^{2}\right]+g_{c} \int d x$ $\cos \left(\sqrt{8} \hat{\phi}_{-}\right)$. The normal modes' velocities $v_{ \pm}$are defined as

$$
v_{ \pm}^{2}=v^{2}\left(1 \pm \frac{K g_{x}}{v}\right) \text {. }
$$

As pointed out by Cazalilla and Ho in Ref. [24], the coupled system (2) is unstable when $v_{-}^{2}$ becomes negative. In other words, the Hamiltonian is not anymore definite positive, pretty much like in the mean-field argument sketched before. This will happen whenever $K g_{x}>v$. Thus, to compute the transition point, we just need to find the Luttinger parameters from the original Hamiltonian (1). In Ref. [24], $K, g_{x}$, and $v$ were approximated via expressions valid in the weak interspecies coupling $g$ regime. In the quasi-condensate regime $\gamma=c / \rho \lesssim 1$ [33], the instability is estimated to happen at $g_{*}=2 c(1-\sqrt{\gamma} / 2 \pi)$. We stress that this result deviates from the mean field value $g_{*}=2 c$.

The phase separation has also been studied analytically beyond perturbation theory by Kolezhuk [26]. He found that for $1 \mathrm{D}$ and two-dimensional gases, the transition point, in the symmetric case, does not depend on the particle densities. Surprisingly enough, the nonperturbative result coincides with the mean-field description; that is, the two species demix when $g \geqslant g_{*}=2 c$. Following this result, one might be tempted to think that the bosonization framework is not able to predict correctly the transition point. It could be argued that the
Luttinger liquid paradigm breaks down at intermediate values of $g$, below the critical value $g_{*}=2 c$. Here we will show that this is not the case. We demonstrate that the bosonization predicts the transition correctly when the Luttinger parameters are computed exactly instead of using approximations.

\section{CMPS SOLUTION}

A translational invariant cMPS of $N$ bosonic species is defined by the state vector [17] ( $N=2$ in this work):

$$
|\chi\rangle=\operatorname{Tr}_{\mathrm{aux}} \mathcal{P} \exp \left[\int_{0}^{L} d x \widetilde{Q} \otimes \mathbb{I}+\sum_{\alpha=1}^{2} \widetilde{R}_{\alpha} \otimes \hat{\psi}_{\alpha}^{\dagger}(x)\right]|\Omega\rangle,
$$

where $\hat{\psi}_{\alpha}(x)$ are the bosonic field operators, $\widetilde{Q}$ and $\widetilde{R}_{\alpha}$ are a set of complex, $\widetilde{D} \times \widetilde{D}$ matrices acting on an auxiliary $\widetilde{D}$ dimensional space, and $|\Omega\rangle$ is the free vacuum state vector $\left(\hat{\psi}_{\alpha}(x)|\Omega\rangle=0\right) . \mathcal{P}$ denotes a path-ordering prescription, and the partial trace, $\operatorname{Tr}_{a u x}$, is taken over the auxiliary space. This way of writing field states is the continuous limit of a MPS [7]. Here the dimension $\widetilde{D}$ of the auxiliary matrices corresponds to the so-called bond dimension, an upper bound to the entanglement entropy. Typically the low-energy states of local Hamiltonians should possess a low amount of entanglement, consequently $\widetilde{D}$ is a small number. If the bond dimension is small, the state (4) represents an efficient trial for finding the ground state of 1D field theories numerically.

In a previous work [18] the authors showed how to construct a two-species cMPS starting from two decoupled single species solutions. In brief, for coupled fields we considered coupled auxiliary spaces (one per bosonic field). The total auxiliary Hamiltonian was extended to $\left(\widetilde{K}=-i \widetilde{Q}-\frac{1}{2} \sum \widetilde{R}_{\alpha}^{\dagger} \widetilde{R}_{\alpha}\right)$ :

$$
\widetilde{K}=K_{1} \otimes \mathbb{I}_{2}+\mathbb{I}_{1} \otimes K_{2}+\sum_{p=1}^{P} Z_{1}^{(p)} \otimes Z_{2}^{(p)}
$$

where $K_{\alpha}$ is the auxiliary Hamiltonian associated to bosonic species $\alpha$. The parameter $P$ accounts for the number of pairs of coupling matrices entering in the cMPS state. Consequently the matrices $\widetilde{R}_{\alpha}$, belonging to the auxiliary space of field $\alpha$, were extended into the total product space: $\widetilde{R}_{1}=R_{1} \otimes \mathbb{I}$ and $\widetilde{R}_{2}=\mathbb{I} \otimes R_{2}$. Denoting $D$ the dimension of the matrices $R_{1}$ and $R_{2}$, the bond dimension is then $\widetilde{D}=D^{2}$. The total number of variational parameters is $D^{2}(4+2 P)$. Details can be found in Ref. [18].

In the thermodynamic limit $(L \rightarrow \infty)$, the fluctuations and correlation functions can be computed from

$$
\begin{aligned}
C_{\alpha \beta}(x-y) & \equiv\left\langle\hat{\psi}_{\alpha}^{\dagger}(x) \hat{\psi}_{\beta}^{\dagger}(y) \hat{\psi}_{\beta}(y) \hat{\psi}_{\alpha}(x)\right\rangle \\
& =\operatorname{Tr}\left[\left(\widetilde{R}_{\beta} \otimes \widetilde{R}_{\beta}^{*}\right) \mathrm{e}^{T(x-y)}\left(\widetilde{R}_{\alpha} \otimes \widetilde{R}_{\alpha}^{*}\right)\right]
\end{aligned}
$$

without loss of generality, we have assumed that $x>y$. Keep in mind that throughout this work \langle\rangle denotes an average over the ground state of (1). The transfer operator $T$ is defined as $T \equiv \widetilde{Q} \otimes \mathbb{I}+\mathbb{I} \otimes \widetilde{Q}^{*}+\sum_{\alpha=1}^{2} \widetilde{R}_{\alpha} \otimes \widetilde{R}_{\alpha}^{*}$. Finally, the fluctuations are calculated by making $x=y$ in (6). 


\section{RESULTS}

As already anticipated, our goal is to characterize the mixing-demixing transition numerically. We do it in two ways. First, we study the instability in the low-energy regime described by the effective Hamiltonian (2). The second strategy is to look directly at the ground state of (1) and compute the fluctuations and correlation functions; cf. Eq. (6).

\section{A. Bosonization instability}

In the harmonic fluid approach the normal modes for the fields decouple [see the discussion below Eq. (2)]. Each of these modes propagate with different velocities, $v_{ \pm}$. Within the bosonization framework, these velocities can be related to the ground state energy density $\left(e_{0}\right)$. The explicit expressions for the velocities are [33,34]

$$
v_{ \pm}^{2}=2 \rho_{ \pm} \frac{\partial^{2} e_{0}}{\partial \rho_{ \pm}^{2}}
$$

with $\rho_{ \pm}=\rho_{1} \pm \rho_{2}$. Analytical estimations for these velocities follow from Eq. (3). In the weak-coupling regime $(g \ll c)$, it is safe to assume that $v$ and $K$ correspond to the solutions for a single bosonic field [33]. In turn, $g_{x}$ is approximated by $g_{x} \simeq g / \pi$ (see Ref. [24]).

In the inset of Fig. 1, it can be seen that already at intermediate values of $g$ (well below the critical value $g_{*}$ ), the predicted velocities $v_{ \pm}$using weak-coupling analytical expressions deviate from the numerically computed ones $[18,34]$. A consequence of this deviation is the failure on the estimation of the point where $v_{-}^{2}$ becomes negative, which in turn marks the critical value $g_{*}$. In Fig. 1 we have zoomed the $v_{-}^{2}$ around the transition point for different values of $\gamma=c / \rho$. As has been already pointed out, within the weakcoupling treatment, the transition is estimated to happen at $g_{*}=2 c(1-\sqrt{\gamma} / 2 \pi)$. As $\gamma=c / \rho$, the latter result makes the transition point dependent on both the intraspecies coupling $c$

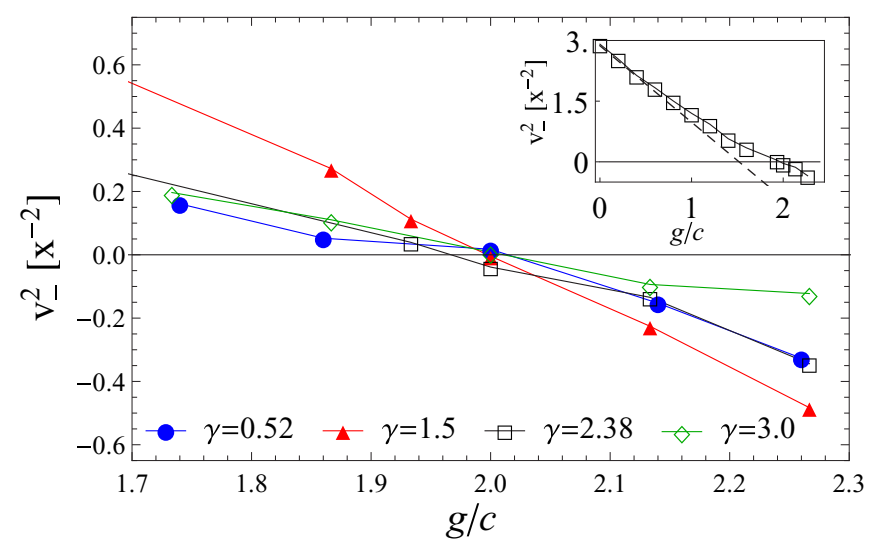

FIG. 1. (Color online) Instability in the bosonization description. The square velocities $v_{-}^{2}$, defined by (7), are calculated using cMPS for different values of the parameter $\gamma=c / \rho$ : 0.52 (filled circles), 1.5 (filled triangles), 2.38 (open squares), and 3.0 (open diamonds). In the inset, we compare the numerical result for $\gamma=2.38$ (open squares) with a weak-coupling estimation (dashed line) for the same value of $\gamma$ with $c=1.5$ and $\rho=0.63$ (see the main text). All of the simulations have been performed with $D=5$ and $P=1$.

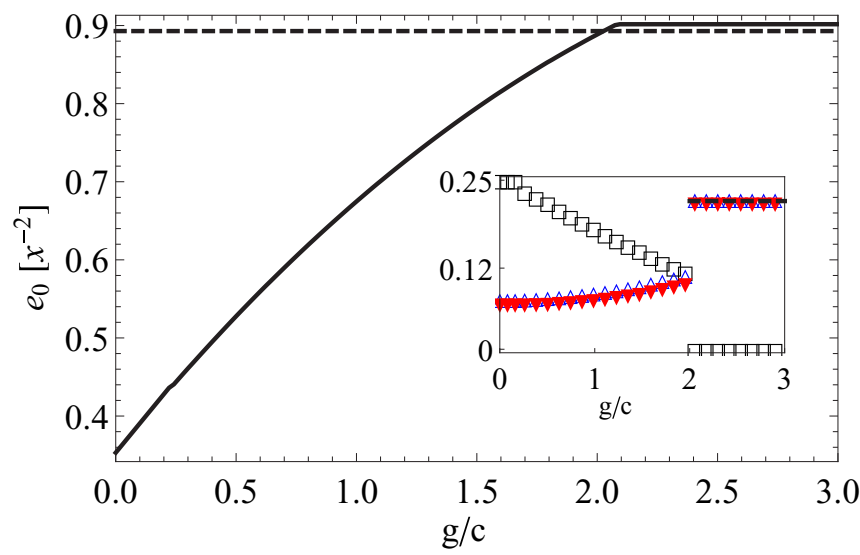

FIG. 2. (Color online) Ground state energy density of Hamiltonian (1) as a function of the coupling ratio $g / c$ calculated with cMPS. We keep fixed the intraspecies coupling $c=1.5$ while the particle density in each of the gases is equal to $\rho=0.5$. In the inset we show the ground state fluctuations as a function of $g / c: C_{11}(0)$ (open triangles), $C_{22}(0)$ (inverted filled triangles), and $C_{12}(0)$ (open squares) defined in (6). Simulations have been performed with $D=5$ and $P=1$.

and the particle density $\rho$. On the other hand, once $v_{-}$is exactly derived from the ground-state energy density by using relation (7), we see how the transition point becomes independent of $\gamma$. In fact, we see that the mode propagating with velocity $v_{-}$becomes ill-defined at $g_{*} / c=2$ [35], in agreement with the mean-field and Kolezhuk results [26]. Therefore, once the Luttinger parameters are exactly computed, the bosonization predicts correctly the transition.

\section{B. Characterization beyond bosonization}

Having a full knowledge of the ground state of (1), we proceed now to characterize the phase transition beyond the bosonization formalism. In Fig. 2, we see the behavior of the ground state energy density as a function of the interspecies coupling. It is direct to realize that after $g_{*} / c=2$ the energy remains constant. In this region, the ground state is such that the last term of (1), i.e., the one accounting for the interaction among different fields, has a zero average. In other words, after the transition we have that $C_{1,2}(0)=$ $\left\langle\hat{\psi}_{1}^{\dagger}(x) \hat{\psi}_{1}(x) \hat{\psi}_{2}^{\dagger}(x) \hat{\psi}_{2}(x)\right\rangle=0$, which is explicitly represented in the inset of Fig. 2 (open squares). This confirms our previous exposition for the phase transition: in one dimension, phase separation implies zero interspecies fluctuations.

Apart from the transition point estimation and the zero field-field overlapping nature for the demixed phase, we can go further in characterizing the properties of the ground state before and after the transition. Let us start with the mixed phase. From the inset of Fig. 2 it follows that the fluctuations $C_{1,2}(0)$ do not remain constant as soon as the interaction is switched on. The latter behavior reflects a sublinear growth of $e_{0}$ in terms of $g$. This means that a simple mean field theory $\left\langle\hat{\psi}_{1}^{\dagger}(x) \hat{\psi}_{1}(x) \hat{\psi}_{2}^{\dagger}(x) \hat{\psi}_{2}(x)\right\rangle \cong \rho_{1} \rho_{2}$ is not sufficient for describing this phase.

We will discuss now the demixed phase. As explained above, after the transition $C_{1,2}(0)=0$. It is straightforward 

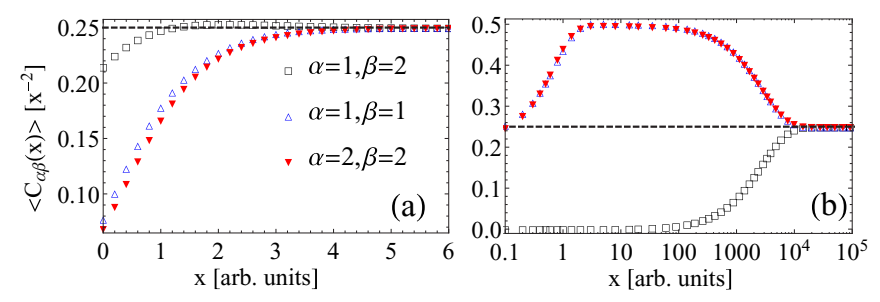

FIG. 3. (Color online) Density correlation functions: $C_{11}(x)$ (open triangles), $C_{22}(x)$ (inverted filled triangles), and $C_{12}(x)$ (open squares) as a function of the distance $x$ for the same parameters of Fig. 2. The transition happens at $g_{*} / c=2$. We plot the correlations (a) before the transition $g / c=0.52$ and (b) after the transition $g / c=2.53$. The shape of this curve brings to mind the popular story of the boa constrictor digesting an elephant [36]. Simulations have been performed with $D=5$ and $P=1$.

to see that a ground state of the form

$$
\left|X_{\mathrm{dm}}\right\rangle=\frac{1}{\sqrt{2}}\left(\left|\chi_{2 \rho}\right\rangle \otimes|\Omega\rangle+\mathrm{e}^{i \theta}|\Omega\rangle \otimes\left|\chi_{2 \rho}\right\rangle\right)
$$

fulfills this condition (the subscript dm stands for demixing). In addition $\left|X_{\mathrm{dm}}\right\rangle$ must satisfy the particle density conservation for each bosonic species: $\left\langle X_{\mathrm{dm}}\left|\hat{\psi}_{\alpha}^{\dagger}(x) \hat{\psi}_{\alpha}(x)\right| X_{\mathrm{dm}}\right\rangle=\rho$, which in turn imposes that $\left\langle\chi_{2 \rho}\left|\hat{\psi}_{\alpha}^{\dagger}(x) \hat{\psi}_{\alpha}(x)\right| \chi_{2 \rho}\right\rangle=2 \rho$. Indeed, this is confirmed in Fig. 2 via the ground state energy density. We see that after the transition, $e_{0}$ is the energy of a single bosonic gas (Lieb-Liniger model) with self-interaction $c$ but double particle density $2 \rho$ (dashed line) [35]. Finally, by looking at the fluctuations $C_{\alpha \alpha}(0)$, we check that they coincide with those of a single bosonic gas with self-interaction $c$ and particle density $2 \rho$, divided by a factor of two due to normalization in (8). The fluctuations of a single gas are shown in the inset of Fig. 2 with a dashed line.

We finish our phase characterization by studying the correlation functions, $C_{\alpha \beta}(x)$. The results are plotted in Fig. 3. By definition, the correlations at zero distance match the fluctuations. On the other hand, in the limit $x \rightarrow \infty$, the correlations factorize yielding $C_{\alpha \beta}(x \rightarrow \infty)=\rho^{2}$ [marked with dashed lines in Figs. 3(a) and 3(b)]. In the mixed phase the correlation length is of the order of $x \cong 5$, pretty much the same as for a single bosonic species with self-interaction $c$ and particle density $\rho$.

More structure for $C_{\alpha, \beta}(x)$ appears in the demixed phase. The interspecies correlation function $C_{12}(x)$, obviously starting at zero, has a large correlation length $\sim 10^{4}$ (notice the logarithmic scale). To understand this large correlation length we recall that after the transition the fields are infinitely repelled. Our interpretation is reinforced by looking at $C_{\alpha \alpha}(x)$. In the range $0<x<10$ the correlations build up to $2 \rho^{2}$, which means that they can be approximated by $C_{\alpha \alpha}(x) \cong$ $1 / 2\left\langle\chi_{2 \rho}\left|\hat{\rho}_{\alpha}\right| \chi_{2 \rho}\right\rangle^{2}=2 \rho^{2}$. Therefore, the coherence has been lost at the single field level. However, the fully uncorrelated state will involve the full state $\left|X_{\mathrm{dm}}\right\rangle$ and pretty much like for the $C_{12}(x)$ correlations, the demixed phase is equivalent to an infinite repulsive phase, explaining again the large coherence length to reach the asymptotic limit $C_{\alpha \alpha}(x \rightarrow \infty)=\rho^{2}$.

\section{SUMMARY}

Summarizing, by means of cMPS, we have computed numerically the ground state of two repulsive $1 \mathrm{D}$ bosonic nonconvertible fields. This kind of systems exhibits the so-called mixing-demixing phase transition. We have validated previous analytical results for the transition point. Furthermore, we have demonstrated that this point can be resolved within the Luttinger liquid formalism whenever the effective parameters of the theory are calculated exactly. All this marks a step forward for the cMPS method, here resolving a phase transition in a nontrivial quantum field theory.

\section{ACKNOWLEDGMENTS}

We acknowledge discussions with Juanjo García-Ripoll and Jorge Alda. We also acknowledge support from the Spanish DGICYT under Project No. FIS2011-25167 as well as by the Aragón (Grupo FENOL) and the EU Project PROMISCE.
[1] J. I. Cirac and P. Zoller, Nat. Phys. 8, 264 (2012).

[2] R. Orús, Ann. Phys. 349, 117 (2014).

[3] I. M. Georgescu, S. Ashhab, and F. Nori, Rev. Mod. Phys. 86, 153 (2014).

[4] T. Giamarchi, Quantum Physics in One Dimension, International Series of Monographs on Physics (Clarendon Press, Oxford, 2004).

[5] S. R. White, Phys. Rev. Lett. 69, 2863 (1992).

[6] U. Schollwöck, Ann. Phys. 326, 96 (2011).

[7] F. Verstraete and J. I. Cirac, Phys. Rev. Lett. 104, 190405 (2010).

[8] I. Bloch, J. Dalibard, and W. Zwerger, Rev. Mod. Phys. 80, 885 (2008).

[9] M. A. Cazalilla, R. Citro, T. Giamarchi, E. Orignac, and M. Rigol, Rev. Mod. Phys. 83, 1405 (2011).

[10] X.-W. Guan, M. T. Batchelor, and C. Lee, Rev. Mod. Phys. 85, 1633 (2013).
[11] H. Moritz, T. Stöferle, M. Köhl, and T. Esslinger, Phys. Rev. Lett. 91, 250402 (2003).

[12] T. Kinoshita, T. Wenger, and D. Weiss, Science 305, 1125 (2004).

[13] B. Paredes, A. Widera, V. Murg, O. Mandel, S. Fölling, I. Cirac, G. V. Shlyapnikov, T. W. Hänsch, and I. Bloch, Nature (London) 429, 277 (2004).

[14] S. Trotzky, Y.-A. Chen, A. Flesch, I. P. McCulloch, U. Schollwöck, J. Eisert, and I. Bloch, Nat. Phys. 8, 325 (2012).

[15] D. Draxler, J. Haegeman, T. J. Osborne, V. Stojevic, L. Vanderstraeten, and F. Verstraete, Phys. Rev. Lett. 111, 020402 (2013).

[16] J. Haegeman, D. Draxler, V. Stojevic, J. I. Cirac, T. J. Osborne, and F. Verstraete, arXiv:1501.06575 [quant-ph]

[17] J. Haegeman, J. I. Cirac, T. J. Osborne, and F. Verstraete, Phys. Rev. B 88, 085118 (2013). 
[18] F. Quijandría, J. J. García-Ripoll, and D. Zueco, Phys. Rev. B 90, 235142 (2014).

[19] S. S. Chung, K. Sun, and C. J. Bolech, Phys. Rev. B 91, 121108 (2015).

[20] G. Modugno, M. Modugno, F. Riboli, G. Roati, and M. Inguscio, Phys. Rev. Lett. 89, 190404 (2002).

[21] T. Schweigler, V. Kasper, S. Erne, B. Rauer, T. Langen, T. Gasenzer, J. Berges, and J. Schmiedmayer, arXiv:1505.03126 [cond-mat.quant-gas]

[22] S. B. Papp, J. M. Pino, and C. E. Wieman, Phys. Rev. Lett. 101, 040402 (2008).

[23] E. Timmermans, Phys. Rev. Lett. 81, 5718 (1998).

[24] M. A. Cazalilla and A. F. Ho, Phys. Rev. Lett. 91, 150403 (2003).

[25] T. Mishra, R. V. Pai, and B. P. Das, Phys. Rev. A 76, 013604 (2007); F. Zhan and I. P. McCulloch, ibid. 89, 057601 (2014).

[26] A. K. Kolezhuk, Phys. Rev. A 81, 013601 (2010).
[27] Y.-L. Lee and Y.-W. Lee, J. Phys. Soc. Jpn. 80, 044003 (2011). [28] D. M. Larsen, Ann. Phys. 24, 89 (1963).

[29] E. V. Goldstein and P. Meystre, Phys. Rev. A 55, 2935 (1997).

[30] P. Ao and S. T. Chui, Phys. Rev. A 58, 4836 (1998).

[31] F. D. M. Haldane, J. Phys. C: Solid State Phys. 14, 2585 (1981).

[32] F. D. M. Haldane, Phys. Rev. Lett. 47, 1840 (1981).

[33] M. A. Cazalilla, J. Phys. B: At. Mol. Opt. Phys. 37, S1 (2004).

[34] A. Kleine, C. Kollath, I. P. McCulloch, T. Giamarchi, and U. Schollwck, New J. Phys 10, 045025 (2008).

[35] The dispersion in the estimation of $g_{*}$ shown in the paper is around $2 \%$. This agrees with the accuracy in the estimation for $e_{0}$; cf. Fig. 2. In addition, errors can also be estimated for the energy of the ground state in the single Lieb-Liniger model as a function of $D$. Again, for $D=5$, it is of the order of $1 \%$.

[36] A. de Saint-Exupéry, The Little Prince (Reynal \& Hitchcock, New York, 1943). 